\title{
STEVE MURPHY , Complexité d'un cœur simple
}

\section{Maria Emanuela Raffi}

\section{OpenEdition \\ Journals}

\section{Edizione digitale}

URL: https://journals.openedition.org/studifrancesi/15678

DOI: 10.4000/studifrancesi. 15678

ISSN: 2427-5856

\section{Editore}

Rosenberg \& Sellier

\section{Edizione cartacea}

Data di pubblicazione: 1 décembre 2018

Paginazione: 518-519

ISSN: 0039-2944

\section{Notizia bibliografica digitale}

Maria Emanuela Raffi, «STeVe muRPHY, Complexité d'un cœur simple», Studi Francesi [Online], 186 (LXII I

III) | 2018, online dal 01 janvier 2019, consultato il 09 novembre 2021. URL: http://

journals.openedition.org/studifrancesi/15678; DOI: https://doi.org/10.4000/studifrancesi. 15678

Questo documento è stato generato automaticamente il 9 novembre 2021.

\section{(c) (†)}

Studi Francesi è distribuita con Licenza Creative Commons Attribuzione - Non commerciale - Non opere derivate 4.0 Internazionale. 


\title{
STEVE MURPHY, Complexité d'un cœur simple
}

\author{
Maria Emanuela Raffi
}

\section{NOTIZIA}

STEVE MURPHY ,Complexité d'un cœur simple, Genève, La Baconnière, 2018, 397 pp.

1 Dopo un «Avant-propos» in cui tutti i tranelli posti dalla semplicità esibita, dalla apparente distanza rispetto alla Storia, dall'ironia con cui Flaubert accompagna questo suo nuovo «livre sur rien» sono rivelati e per ciò stesso un po' aggirati, Murphy dichiara di dedicarsi alla lettura «de l'implicite» che costituisce in realtà l'ossatura di Un Cour simple.

2 Nei successivi quattordici capitoli, completati da un'ampia e particolareggiata bibliografia, Murphy conduce con la consueta vivacità di scrittura un'analisi minuziosa del conte flaubertiano - in un va e vieni continuo dal testo ai brouillons - e dei giudizi e pregiudizi critici che lo accompagnano, a cominciare dalla sua presunta "astoricità», mostrando la presenza «d'une forme d'historicité plus diffuse, avec des coordonnées socilogiques et idéologiques» indubbiamente sfumate, ma non meno strutturanti («L'Histoire souterraine»). Ne fa parte anche la collocazione sociale di Mme Aubain, sospesa fra "petite noblesse» e «bourgeoisie», dotata di un unico parente nobile poco gratificante, il marchese de Gremanville «ruiné par la crapule», e di un marito «beau garçon sans fortune», muscadin morto giovane. E attorno a questa figura femminile centrale, è tutto il «micro-univers mortifère de la maison Aubain» che Murphy passa in rassegna-abitanti, visitatori, ambienti, oggetti, animali-nella loro progressiva decadenza («Questions de classe»).

Un cambio di prospettiva appare nel terzo capitolo («Théodore n'est pas un cadeau»), dove è preso in considerazione il personaggio del giovane e ambiguo spasimante di Félicité, Théodore, "parodie du héros bonapartiste» che cerca di evitare con tutti i mezzi la chiamata alle armi. È poi la volta di Paul e Virginie (nomi letterariamente 
«surdéterminés»), attraverso i quali Murphy rileva sia la penosa situazione dei bambini legati da Flaubert alla patologica esistenza di Mme Aubain, sia gli esiti anch'essi patologici della loro vita di adulti. Paul, copista, «personnage falot dont l'authenticité est celle de la copie d'une copie» e Virginie fragile e malata, morta vergine in convento, portatrice di una serie intricata di rinvii letterari: al personaggio di Bernardin de SaintPierre e al capitano della nave Aubin, che compongono insieme Virginie Aubain («au bain, toujours...», a sua volta rinvio all'iconografia del genere Suzanne au bain...).

«L'amour de Victor», quinto capitolo, è dedicato al nipote di Félicité, ai suoi piccoli «cadeaux» e alla possibilità di sognare viaggi e avventure che regala alla zia con i suoi racconti. Infine, e soprattutto, alla sua morte, occasione per Flaubert per denunciare ancora una volta l'impreparazione della classe medica (qui nel 1820) e l'inutilità delle sue terribili «saignées». Il quadro analitico e critico in cui, pagina dopo pagina, Murphy mostra il complesso intreccio di riferimenti intertestuali, soprattutto con Madame Bovary, e più in generale storico-letterari di Un cœur simple, si arricchisce nel capitolo successivo del personaggio di Bourais, "parodie ambulante des valeurs de Diderot et de Voltaire», parassita che fonda le sue azioni sul suo «manque de cœur» («Bourais assassin ou le QI du cuistre»).

«Le baromètre existentiel» segna uno spostamento di interesse verso la centralità del détail spesso rimproverata a Flaubert come fattore d'impoverimento. Partendo dalla lettura di Barthes e al ben noto «effet de réel» che attribuisce al «baromètre de Flaubert», Murphy traccia, muovendosi nell'ampia bibliografia critica sugli oggetti flaubertiani, un altro percorso, in cui il barometro di Mme Aubain «prend sens»; esso allude infatti sia al tempo metereologico, che al «temps existentiel suspendu entre la non-vie d'une depression et la mort qui plane».

6 I due capitoli successivi («La résilience d'une femme en bois» e «Politiques de Félicité?») mettono quasi l'una di fronte all'altra le due figure femminili del romanzo, legate da mezzo secolo di convivenza, ma separate da una evidente disparità sociale ed economica. La differenza fra le due donne appare particolarmente evidente alla prova del dolore per la morte di Victor e di Virginie: mentre Félicité tende a controllare i propri sentimenti in modo che «son chagrin et un moment de colère laissent la place à l'activité domestique» in una specie di automatismo ripetitivo, Mme Aubain si ripiega su se stessa «assise sur sa chaise comme un perroquet muet perché dans sa cage». Riunite per un attimo dal dolore per la morte di Virginie in un «baiser faussement égalisateur», specchio dell'«illusion fraternitaire» prodotta dagli eventi del 1830 e poi del 1848 , le due donne mantengono per tutto il romanzo un «lien quasi féodal», que Félicité sostiene e giustifica anche con la sua compassione religiosa.

7 Loulou, il pappagallo di Félicité, unica emanazione del 1830 realmente presente secondo Murphy nel romanzo, occupa i tre capitoli successivi («Ceux par qui Loulou arrive», «Loulou vivant, mort et revenant» e «Félicité au bout du chemin»). La sua origine, dono del barone di Larsonnière consegnato da un servitore nero, porta con sé il dibattito sulla schiavitù e sulla libertà concluso nella seconda Repubblica e ancora di grande attualità nel continente americano. Murphy mette in relazione, con approfondimenti spesso ironici, «la pauvreté de langage» di Félicité e la sobrietà cromatica dei suoi vestiti con l'ignoranza linguistica del pappagallo, che paradossalmente è proprio la domestica a istruire, e i bellissimi colori del piumaggio. La simbiosi fra $\mathrm{i}$ due, "cette espèce d'union sublimée» che mostra qualche valenza sessuale, ma al tempo stesso altamente spirituale («le Saint-Esprit»), è conclusa dalla 
morte del pappagallo soffocato (dal fumo, dalla mancanza di libertà, dall'amore soffocante di Félicité, dalle pulsioni autodistruttive di Loulou?) e dalla sua resurrezione come animale impagliato. Alla morte di Félicité, «par le truchement du reposoir, Loulou pourra enfin quitter sa branche d'acajou pour prendre son glorieux envol», ma non senza lasciare ancora un inquietante legame con la sua padrona e perfino con Mme Aubain: «Sur le plan symbolique, la maison Aubain serait le lieu d'une crise épidémique de la répétition qui se poursuivrait jusque dans les circonstances des morts».

8 Nei due ultimi capitoli («Le bazar, la chapelle et des fissures» e «Champs de vision: le monde du spectral») i luoghi e gli oggetti di Un cour simple arrivano alla ribalta, a partire dal bazar di Félicité, «mise en abyme du "mausolée" qu'est devenue la maison entière de Mme Aubain», a sua volta oggetto "d'une métaphorisation qu'apparente la maison à une tête, les fenêtres aux yeux et les persiennes aux paupières». Una casa, inoltre, attraversata e visitata da visioni e fantasmi che culminano nell'immagine del pappagallo gigante «planant au-dessus de la tête de Félicité» in veste di Spirito Santo, trasformazione che può avvenire per Murphy solo a certe condizioni: «pour Félicité l'advenue du merveilleux ne peut se comprendre en dehors de la privation sensorielle qui la plonge dans une intense désorientation et même dans un monde à part» $\mathrm{e}$ nemmeno al di fuori di una forte connotazione religiosa.

9 Con la morte di Félicité si conclude il densissimo percorso di Murphy attraverso il racconto flaubertiano, rilettura critica consapevole di innumerevoli contributi, che tuttavia non vuole mostrare il racconto come precipitazione verso la catastrofe sociale ed emotiva: «Un coeur simple n'est pas réductible à une histoire de mort: c'est aussi, et avant tout, une glorification de la résistance à l'adversité et une célébration des valeurs de la vie». 